\title{
When to Introduce Three-Dimensional Visualization Technology into Surgical Residency: A Randomized Controlled Trial
}

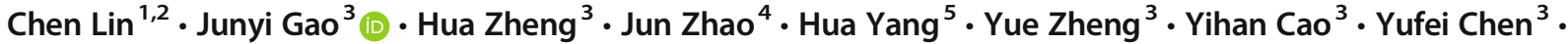 \\ Guoliang $\mathrm{Wu}^{3}$. Guole Lin ${ }^{1}$. Jianchun $\mathrm{Yu}^{1}$ • Hanzhong $\mathrm{Li}^{6}$ • Hui Pan ${ }^{2,4}$ • Quan Liao ${ }^{1}$ Y Yupei Zhao ${ }^{1}$
}

Received: 14 September 2018 / Accepted: 8 January 2019 / Published online: 9 February 2019

(C) The Author(s) 2019

\begin{abstract}
Three-dimensional (3D) reconstructed images have been increasingly applied for medical education. Although many studies have described the benefits of such applications, the best time to introduce 3D technology into surgical training has not been determined. Therefore, we conducted a randomized study to determine a suitable period for the introduction of this technology. Seventy-one surgical residents were randomized into 2 groups (two-dimensional computed tomography (CT) group and 3D image group), and they completed a test on anatomy and imaging as well as a questionnaire. Post-graduate year 1 (PGY1) residents in the 3D group performed significantly better than those in the CT group, although the third-year residents did not present significant differences in either the score or the time spent answering the questions. Although residents in different years of training held different attitudes toward the difficulty of anatomy and imaging learning, they all showed a high level of acceptance of the 3D training. This study revealed that 3D images improved the junior residents' performance in imaging reasoning. Thus, systematically introducing 3D images early in a surgical resident training program may help produce a better anatomy-imaging-surgery system.
\end{abstract}

Keywords Residency training $\cdot 3 \mathrm{D}$ technology $\cdot$ Anatomy-imaging-surgery system

With the rapid development of digital technologies, threedimensional visualization systems have shown increasing potential value in medical education. A series of studies indicated that 3D anatomy models have been widely used in surgical teaching, patient education and operation planning [1-3] and

Chen Lin and Junyi Gao These authors contributed equally to this work.

This article is part of the Topical Collection on Education \& Training

Electronic supplementary material The online version of this article (https://doi.org/10.1007/s10916-019-1157-0) contains supplementary material, which is available to authorized users.

Quan Liao

lqpumc@126.com

Yupei Zhao

zhao8028@263.net

1 Department of General Surgery, Peking Union Medical College Hospital (PUMCH), Chinese Academy of Medical Sciences \& Peking Union Medical College (CAMS \& PUMC), Beijing, China

2 National Virtual Simulation Laboratory Education Center of Medical Sciences, PUMCH, CAMS \& PUMC, Beijing 100730, China that these models have many advantages over traditional methods, especially in complex anatomy, such as the larynx and inner ear $[4,5]$.

Standardized training of residents has become a core issue in medical education in China in recent years, and a method of
3 Eight-year Program of Clinical Medicine, PUMCH, CAMS \& PUMC, Beijing, China

4 Department of Education, PUMCH, CAMS \& PUMC, Beijing 100730, China

5 Department of Head and Neck Surgery, PUMCH, CAMS \& PUMC, Beijing 100730, China

6 Department of Urology Surgery, PUMCH, CAMS \& PUMC, Beijing 100730, China 
enhancing the effects of surgical training within a limited time must be identified. Although many studies have suggested the benefits of combining medical teaching with $3 \mathrm{D}$ technologies $[6,7]$ a $3 \mathrm{D}$-based systematic training program for residents is lacking, and few studies have described the best period for the introduction. Thus, we conducted a randomized controlled study to assess the impact of $3 \mathrm{D}$ reconstruction images on the identification of abnormal results and to determine the best period to introduce $3 \mathrm{D}$ technology into resident training programs.

\section{Methods}

\section{Generating 3D simulation models with 3D technology}

The 3D multi-touch visualization touchscreen table (MVT) is a large multi-touch medical table introduced by Sectra in 2010 at the Radiological Society of North America. It is approachable for medical students and surgeons for its high interactivity and short learning curve. Teachers can use the builtin cases to create real-time $3 \mathrm{D}$ reconstructions, and this table includes connections between the cross-sectional CT and 3D images for better medical education.

In this study, raw CT data of nine built-in cases, including two anatomical cases and seven surgical cases, were assessed, and the diseases presented in these cases included bone fracture, aneurysm, ureteral calculi, splenic rupture, pheochromocytoma and hiatal hernia. Then, 3D images were reconstructed with these CT data by the MVT. All of the 3D images were checked and assessed by two attending doctors from the surgical department of Peking Union Medical College Hospital (PUMCH), and they were used in the following tests.

\section{Randomized grouping}

All residents participating in this study were from the surgical department of PUMCH and assigned to several groups by stratified randomization. Stratified Randomization was employed on the basis of one factor, namely post-graduate year (PGY), as this may have affected the test results (specifically PGY1, PGY2 and PGY3\&4 residents constituted the stratification groups). Regarding technical details, Zelen's algorithm was used by an invited staff from the Education Department of our center [8]. The invited staff had no conflicts of interest, did not participate further in the study and signed a Confidentiality Agreement as appropriate.

\section{Imaging test and questionnaire}

The tests consisted of 2 anatomical cases and 7 surgical cases and were developed by a group of attending doctors in the surgical field, who believe the questions are appropriate for assessing the residents' surgical imaging reasoning abilities. The brief medical histories and questions were nearly identical for both the 3D and 2D groups except that the 3D reconstructed images and original CT images from the same cases were provided to the groups (as shown in Fig. 1).

Every case had three to four points that corresponded to detailed answers. Possible scores for the test ranged from 0 (all incorrect) to 34 (all correct). Each test paper was graded by an individual blinded to the corresponding type of images. The time each resident spent answering were also recorded by the online test system.

After the test, all residents were requested to complete a questionnaire (Table 1). This questionnaire was designed by a group of experts, based on the questionnaires of the System for the Evaluation of the Teaching Qualities (SETQ) and several studies on subjective measures toward anatomy and simulation-based education [9-11], with the aim to assess the residents' attitudes toward anatomy learning, imaging learning and 3D training. All of these questions were rated on a 5-point Likert-type scale.

\section{Statistical analysis}

The summary statistics were described as the mean \pm standard deviation (SD). A two-sided unpaired Student's $t$ test was applied to evaluate the influence of the 3D images on the sum scores and time spent answering, and the level of statistical significance was set at a $p$ value $<0.05$. Categorical variables were presented as percentage. Statistical analyses were performed using SPSS (version 23.0 for Mac).

\section{Ethical approval}

Ethical approval was obtained from the Institutional Review Board of the Institute of Basic Medical Sciences, Chinese Academy of Medical Sciences (Project No:009-2014). We maintained confidentiality by keeping relevant information in a separate database and only reviewing aggregate data. To protect the privacy of all participants, no identifying information was recorded. In addition, all of the data were stored on a password-protected computer, and the surveys were stored in a locked filing cabinet. All participants completed written informed consent. Study methods were performed in accordance with the approved guidelines.

\section{Results}

\section{Characteristics of residents}

In this randomized controlled study, a total of 71 surgical residents (66 males and 5 females) participated, and there was no attrition. Thirty-six residents, consisting of nine 
Fig. 1 Images of an anatomy case about abdominal vessels in the CT group (a-d) and 3D group (eg). Four points were distributed to the 1-4 anatomical structures marked with orange or blue arrows: splenic artery, common hepatic artery, celiac trunk and superior mesenteric artery

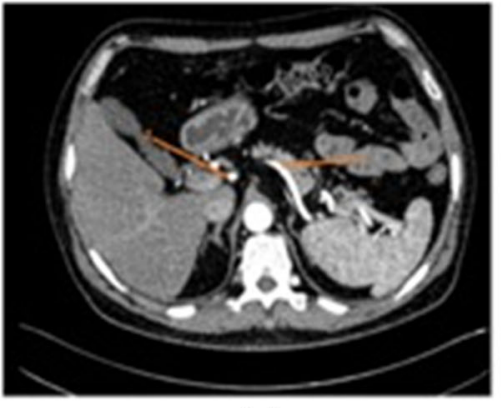

(a)

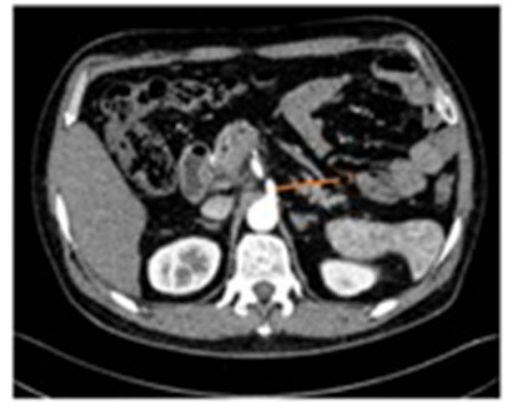

(c)

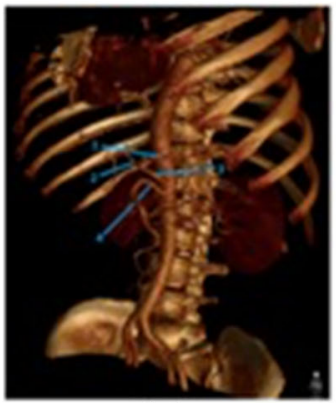

(e)

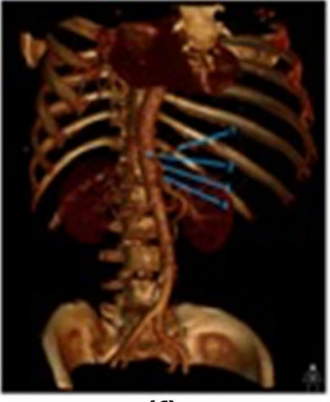

(f)

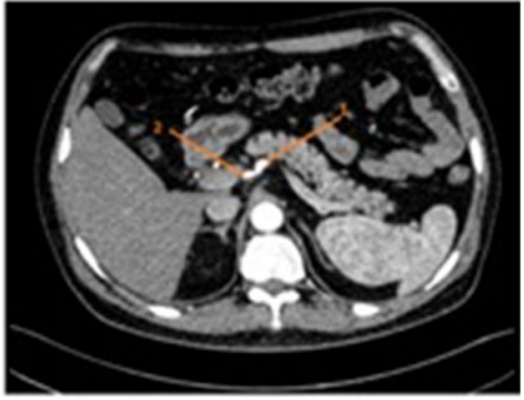

(b)

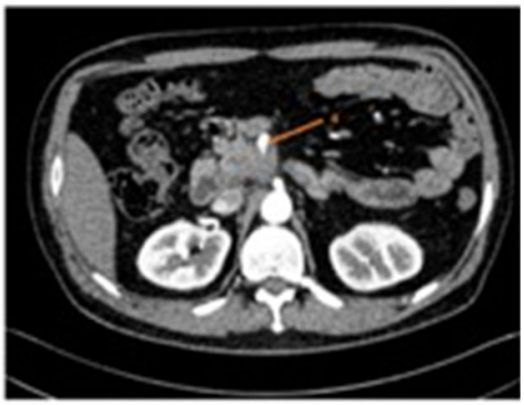

(d)

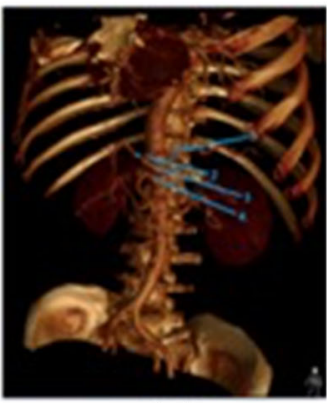

(g)
PGY1, thirteen PGY2, thirteen PGY3 and one PGY4, were randomized into the $3 \mathrm{D}$ group, and thirty-five residents, consisting of eight PGY1, fourteen PGY2, twelve PGY3 and one PGY4, were grouped into the CT group. The gender distribution is detailed in Table 2. In addition, the PGY4 residents were included in the PGY3\&4 group because there is only two PGY4 observed in the sample.

\section{Sum scores of correct answers and time spent}

Overall, the PGY1 and PGY2 sum scores (Fig. 2a) were significantly different between the 3D and CT groups (PGY1, 3D vs. CT: mean difference $(\mathrm{MD})=3.2, p=0.03$. PGY2, 3D vs. $\mathrm{CT}: \mathrm{MD}=3.9, \mathrm{p}<0.001)$, whereas significant differences were not observed in the PGY3\&4 scores between the two groups (3D vs. CT: $\mathrm{MD}=1.5, p=0.11$ ).

An analysis of the time spent answering (Fig. 2b) revealed a significant difference between the two groups of PGY1 residents; the PGY1 residents in the 3D group required $1267 \pm$
$105 \mathrm{~s}$ to complete the test, whereas those in the 2D group required $1455 \pm 157 \mathrm{~s}$ (PGY1, 3D vs. CT: $\mathrm{MD}=-188, p=$ 0.01 ), while there were no differences between the PGY2 and PGY3\&4 residents in the two groups (PGY2, 3D vs. CT: $\mathrm{MD}=-37, P=0.71$. PGY3\&4, 3D vs. $\mathrm{CT}: \mathrm{MD}=-51, p=$ $0.39)$.

\section{Answers to the questionnaire}

The feedback for the questions (Table 1) related to "Learning difficulty" indicated that the PGY1 residents had a more difficult time learning anatomy and imaging reasoning than the PGY3\&4 residents (PGY1 vs. PGY3\&4. Anatomy learning: mean score $=4.65$ vs $3.78, p<0.001$; imaging reasoning learning: mean score $=4.53$ vs. $3.81, \mathrm{p}<0.001)$. When asked about their personal attitude toward these training requirements, the residents in the different training years responded similarly, and the mean scores were all close to 4 points or higher. The entire multiple-choice questionnaire presented 
Table 1 Subjective evaluation questionnaire (1-strongly disagree, 5 -strongly agree) and feedback results. $p$-values obtained via an independent-samples $t$ test performed between groups of PGY1 and PGY3\&4 residents

\begin{tabular}{|c|c|c|c|c|c|}
\hline \multicolumn{2}{|c|}{ Survey Questionnaire for Medical Imaging } & \multicolumn{4}{|c|}{ Feedback results } \\
\hline \multirow[t]{2}{*}{ PGY year: $1 / 2 / 3 / 4$} & \multirow{2}{*}{$\begin{array}{l}\text { Gender: } \\
\quad \text { Male/Female }\end{array}$} & \multicolumn{3}{|c|}{ Mean scores } & \multirow[t]{2}{*}{$P^{*}$} \\
\hline & & PGY1 & PGY2 & PGY3\&4 & \\
\hline \multicolumn{6}{|c|}{ Anatomy and imaging training } \\
\hline $\begin{array}{l}\text { Learning } \\
\text { approaches }\end{array}$ & $\begin{array}{l}\text { 1.1 A.Atlas of anatomy B.Textbooks } \\
\text { C.Reference D.Lectures E.Videos F.3D } \\
\text { form G.Other }\end{array}$ & - & - & - & - \\
\hline \multirow[t]{2}{*}{ Learning difficulty } & 1.2 It is hard to learn anatomy & 4.65 & 4.26 & 3.78 & $<0.001$ \\
\hline & 1.3 It is hard to learn imaging reasoning & 4.53 & 4.30 & 3.81 & $<0.001$ \\
\hline Value for career & $\begin{array}{l}\text { 1.4 It is crucial to strengthen anatomy and } \\
\text { imaging learning for a future surgical } \\
\text { career. }\end{array}$ & 4.00 & 4.26 & 4.19 & 0.43 \\
\hline \multicolumn{6}{|c|}{ 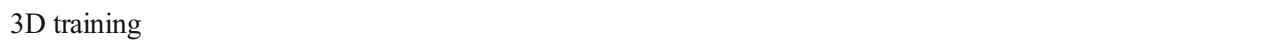 } \\
\hline Simplification & $\begin{array}{l}2.13 \mathrm{D} \text { images make complex anatomy } \\
\text { easier. }\end{array}$ & 3.88 & 3.96 & 4.11 & 0.37 \\
\hline \multirow[t]{2}{*}{ Enjoyment } & $\begin{array}{l}2.23 \mathrm{D} \text { images arouse my interest in } \\
\text { anatomy learning. }\end{array}$ & 4.00 & 4.00 & 4.15 & 0.59 \\
\hline & $\begin{array}{l}2.33 \mathrm{D} \text { images arouse my interest in } \\
\text { imaging learning. }\end{array}$ & 4.06 & 4.00 & 4.19 & 0.50 \\
\hline $\begin{array}{l}\text { Intention to } \\
\text { introduce }\end{array}$ & $\begin{array}{l}2.4 \mathrm{It} \text { is necessary to combine } 3 \mathrm{D} \text { images } \\
\text { with } 2 \mathrm{D} \text { learning during the beginning } \\
\text { of the residency. }\end{array}$ & 4.41 & 4.26 & 4.30 & 0.44 \\
\hline Suggestions & $\begin{array}{l}2.5 \text { What do you think about the } 3 \mathrm{D} \\
\text { training? Any suggestions for future } \\
\text { improvement? (free form) }\end{array}$ & - & - & - & - \\
\hline
\end{tabular}

acceptable reliability and validity, with a Cronbach's alpha of 0.753 and a KMO of $0.710, p<0.001$. In addition, the feedback for the question related to "Learning approaches" revealed that atlas of anatomy and textbooks were the major approaches for the residents to study anatomy, accounting for $85.9 \%$ and $38 \%$ respectively, and other approaches were relatively fewer taken $(21.1 \%$ for videos, $11.3 \%$ for reference and $5.6 \%$ for lectures, and no one used 3D forms).

\section{Discussion}

How to improve the core competence of residents in a more efficient manner is always a standing question for standardized surgical training programs. Surgical education is built on four pillars: surgical theory, clinical surgery, operative techniques and clinical research [12]. Anatomy, the foundation of surgical theory and operative technique, is informative and complex, and it is often the bottleneck of surgery training. In addition, global curriculum reform has led to a reduction in the time and content of anatomical teaching $[12,13]$. This requires us to explore more efficient informational-transfer methods such as 3D technology.

Currently, 3D simulation software is used for surgical education and training, such as learning procedures of laparoscopic pyeloplasty via 3D printing and training orthopaedic residents to drill lateral mass screws via 3D navigation [14, 15]. Although the 3D technology applied in medical education has achieved initial success, it is still not systematically used in surgical residency programs. There were some reports of randomized studies regarding the effect of $2 \mathrm{D}$ vs $3 \mathrm{D}$ teaching modality in the residency. For instance, using 3D printed models of congenital cardiovascular lesions to supplement an educational lecture could improve paediatric medicine residents' scores on a board-style examination [16]; 3D CT may help learning acetabular fracture patterns and correctly applying a widely accepted fracture classification system among both orthopaedic residents and fellows, compared with 2D
Table 2 Baseline characteristics of residents

\begin{tabular}{llllll}
\hline Year of residency training & \multicolumn{2}{l}{ Residents in the 3D CT group, no. (\%) } & & \multicolumn{2}{l}{ Residents in the 2D CT group, no. (\%) } \\
\cline { 2 - 3 } & Male & Female & & Male & Female \\
\hline 1 & $8(88.9)$ & $1(11.1)$ & & $7(87.5)$ & $1(12.5)$ \\
2 & $12(92.3)$ & $1(7.7)$ & & $14(100)$ & $0(0)$ \\
$3 \& 4$ & $13(92.9)$ & $1(7.1)$ & $12(92.3)$ & $1(7.7)$ \\
\hline
\end{tabular}


(a)

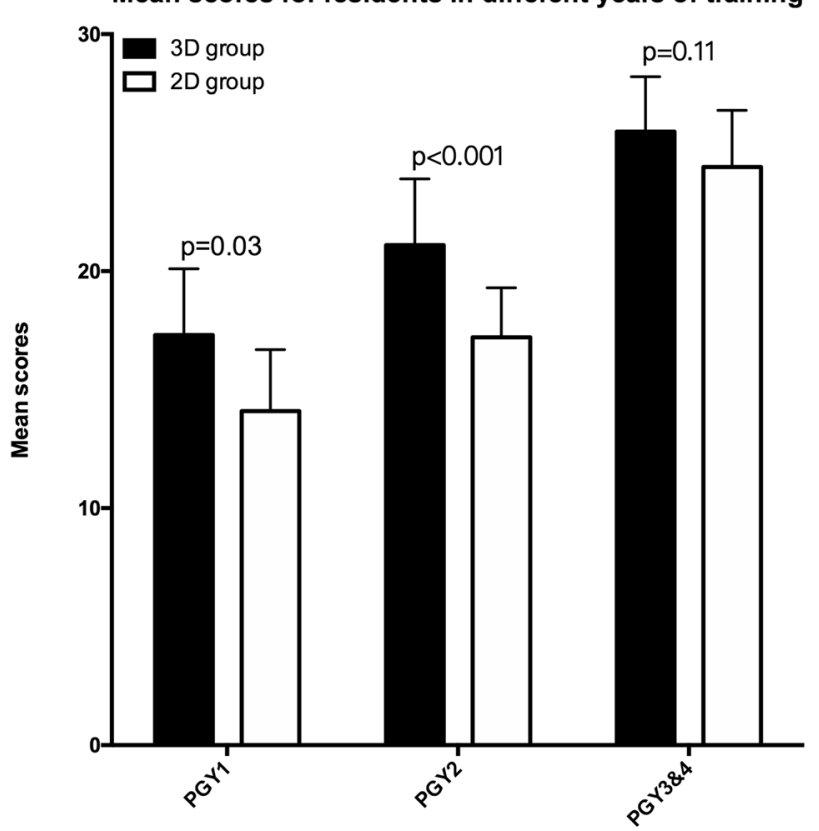

(b) Mean time spent for residents in different years of training

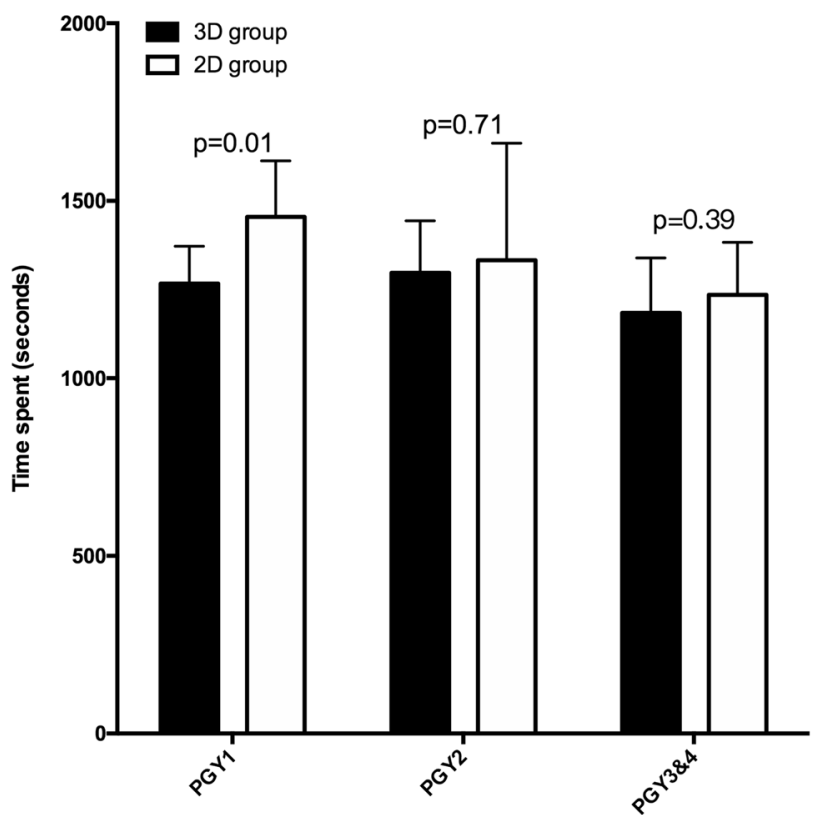

Fig. 2 Mean scores (A) and time spent (B) for residents in different years of training. $p$ value obtained via an independent-samples t-test

axial CT [17]. However, very few studies showed the different attitude, such as Lim's study who compared the role of XR, $\mathrm{CT}$ and 3D models in the accuracy of acetabular fracture identification among orthopaedic residents and concluded 3D models could be an important adjunct to orthopedic residency education but was not significantly superior to identification with CT scans [18]. Additionally, few studies have explored the appropriate introduction time of 3D technology to residency programs; thus, we designed this study to explore the optimal timing for introducing 3D technology into a surgical residency program to improve the trainees' clinical thinking and learning curve.

In both the $2 \mathrm{D}$ and $3 \mathrm{D}$ groups, the PGY3\&4 residents performed significantly better than the PGY1 residents, and the questionnaire results suggested that it was more difficult for PGY1 residents to learn anatomy and imaging. These results are consistent with the medical learning law that clinical thinking gradually improves as experience accumulates. Nevertheless, the PGY3\&4 residents in the 3D group did not show differences from those in the 2D group in the total scores and time spent, which may have been because senior residents had established substantial clinical thinking and spatial imagination. Moreover, different results were observed in the PGY1 residents between the groups. The PGY1 residents in the $3 \mathrm{D}$ group performed significantly better in accuracy and rapidity, and this discrepancy underscored the advantages of 3D images for improving intuition and simplifying complex structures. For beginners at the early stage of the learning curve, such as the PGY1 residents, their clinical thinking is still dependent on informational-transfer methods. Thus, 3D images likely had a superior ability to transfer information among the junior residents.

In the traditional learning model, which could be summarized as " $2 \mathrm{D} \rightarrow 3 \mathrm{D} \rightarrow 2 \mathrm{D}$ " (Fig. 3a), trainees must imagine $3 \mathrm{D}$ structures with $2 \mathrm{D}$ cross-sectional images. This process is long and difficult and often results in the memorization of inaccurate or even incorrect 3D structures because of the lack of an ability to immediately correct these representations. As a result, junior residents will face many problems in the clinic when only $2 \mathrm{D}$ images are available. Our findings suggest that junior trainees may be able to interpret 3D images more easily than 2D images. Accordingly, 3D images might be superior in assisting junior residents in establishing 3D models. This new learning model combines 3D and 2D images and might accelerate the learning process by improving the accuracy of 3D structures and deepening the memory of anatomy. We summarize this learning model as " $2 \mathrm{D}+3 \mathrm{D} \rightarrow 3 \mathrm{D} \rightarrow 2 \mathrm{D}$ " (Fig. $3 b)$. Therefore, we speculate that if this new learning model is systematically introduced into the resident training at PGY1, it may improve the learning curve with respect to the anatomyimaging-surgery system.

Fewer residents considered anatomy and imaging to be difficult as the grade increased. Although anatomy and imaging did not appear to be difficult for senior residents, most of them agreed that it could strengthen their systematic training. This result revealed the importance of anatomy and imaging for surgeons and indicated that a gap occurs between the urgent demand for training and the current insufficiency in training. In addition, 3D images, which present advantages that include improved intuition and simplified anatomy, are considered to increase the trainees' interest in anatomy and imaging learning. Moreover, almost all the residents strongly 
Fig. 3 Imaging learning model. a Classical learning model: " $2 D \rightarrow$ $3 D \rightarrow 2 D$ ". (1) Long and difficult learning process that results in vague $3 \mathrm{D}$ structures due to lack of immediate correction. (2) Process of clinical practice in which residents must correct and rebuild this structure. b New learning model combining 2D and 3D images: " $2 D+3 D \rightarrow 3 D \rightarrow 2 D$ ". (1) Accelerated learning process in which structures can be corrected in both a timely and repeated manner, which results in accurate 3D structures. (2) Process of clinical practice in which the remembered 3D structures are successfully applied (a)
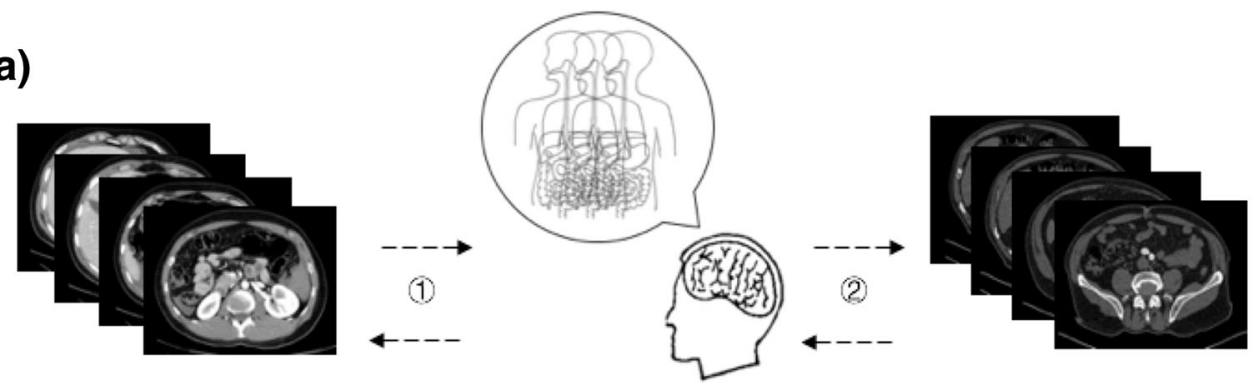

(b)

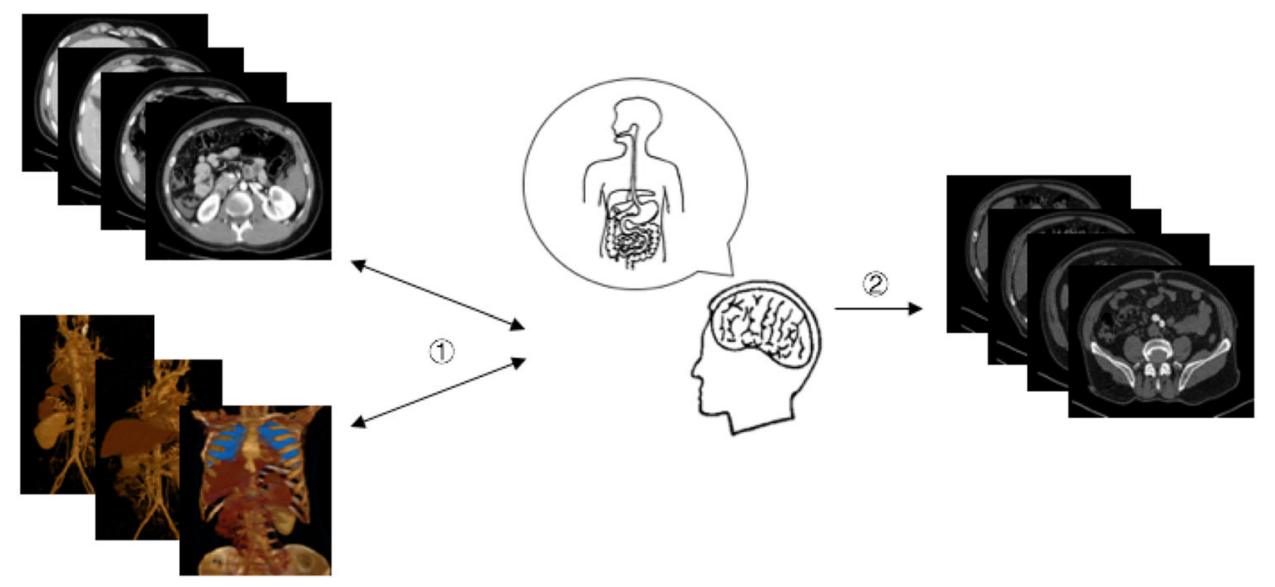

supported the introduction of 3D training into the early stages of residency training.

In addition, residents in our study also encouraged us to introduce $\mathrm{CBL}$ into 3D training for anatomy and imaging. Systematic training with real clinical cases can aid in memorizing and understanding material and reduce the gap between theory and practice.

The study limitations are as follows. Although this is a prospective randomized study, a limited number of participants with few female residents were enrolled in this study. To achieve more accurate results, further studies with larger sample sizes and more females enrolled are needed. Besides, although stratified randomization was employed to ensure that residents at a comparable stage of training were assigned to the intervention and control groups, we could not fully account for the possibility that surgical knowledge level and/or hours spent working on surgical cases similar to those assessed in the test may have differed between the participants. Furthermore, as the test used in this study was developed by surgical experts in a single center, the results should be validated with the use of different standardized tests to increase their generalizability. In addition, the impacts of 3D technologies observed inspired us to design novel standard training courses, in which the combined 2D and 3D technology would be introduced, to further confirm the hypothesis raised in this article.

\section{Conclusion}

This randomized study revealed that 3D images improved junior surgical residents' imaging reasoning performance and were widely accepted and appreciated by all the residents, demonstrating their potential in establishing a more accurate anatomy-imaging-surgery system among young trainees in a more efficient manner. Therefore, it is recommended that combined 3D and 2D training, especially with real clinical cases, be systematically introduced into surgical residency programs early during training.

Acknowledgements The authors appreciate the support from all the surgical residents from PUMCH. This project was supported by grants from funds for Peking Union Medical College Postgraduate Education Reform Program (No. 10023201600101) and funds for Peking Union Medical College Education Reform Program (No. 2015zlgc0126). This work was partly presented at the 24th Annual Meeting of The Society in Europe for the Simulation Applied to Medicine, June 27-29, 2018, Bilbao, Spain.

Author Contributions C.L. and J.G. conducted the study, analysed the results and wrote the paper. H.Y., G.L., J.Z., H.Z., Y.Z., Y.C., Y.C. and G.W. participated in conducting the experiment and collecting data. Y.Z., Q.L., H.P., H.L., J.Y., C.L. and J.G. designed the trial. All authors reviewed the manuscript.

Data availability The datasets generated during and/or analysed during the current study are available from the corresponding author on reasonable request. 


\section{Compliance with ethical standards}

Competing interests The authors declare no competing interests.

Open Access This article is distributed under the terms of the Creative Commons Attribution 4.0 International License (http:// creativecommons.org/licenses/by/4.0/), which permits unrestricted use, distribution, and reproduction in any medium, provided you give appropriate credit to the original author(s) and the source, provide a link to the Creative Commons license, and indicate if changes were made.

Publisher's Note Springer Nature remains neutral with regard to jurisdictional claims in published maps and institutional affiliations.

\section{References}

1. Blum, M. G., and Powers, T. S., Bronchoscopy simulator effectively prepares junior residents to competently perform basic clinical bronchoscopy. Ann. Thorac. Surg. 78(1):287-291, 2004.

2. Yoder, D. M., Fowler, W. C., and Hey, L. A., Anatomic eye models as a patient education modality. Ophthalmic Practice. 19:150-156, 2001.

3. Yang, X. et al., Computer-assisted surgical planning and simulation for condylar reconstruction in patients with osteochondroma. Br. J. Oral Maxillofac. Surg. 49:203-208, 2011.

4. $\mathrm{Hu}, \mathrm{A}$. et al., Evaluation of a three-dimensional educational computer model of the larynx: Voicing a new direction. J. Otolaryngol. Head Neck Surg. 39:315-322, 2010.

5. Nicholson, D. T., Chalk, C., Funnell, W. R., and Daniel, S. J., Can virtual reality improve anatomy education? A randomised controlled study of a computer-generated three-dimensional anatomical ear model. Med. Educ. 40:1081-1087, 2006.

6. Li, Z. et al., Three-dimensional printing models improve understanding of spinal fracture-a randomized controlled study in China. Sci. Rep. 5:11570, 2015.
7. Prinz, A., Bolz, M., and Findl, O., Advantage of three dimensional animated teaching over traditional surgical videos for teaching ophthalmic surgery: A randomised study. Br. J. Ophthalmol. 89:14951499, 2005.

8. Zelen, M., A New Design for Randomized Clinical Trials. NEJM 300(22):1242-1245, 1979.

9. Lombarts, K. M., Bucx, M. J., and Arah, O. A., Development of a system for the evaluation of the teaching qualities of anesthesiology faculty. Anesthesiology 111(4):709-716, 2009.

10. Plaisant, O. et al., Medical students' attitudes towards science and gross anatomy, and the relationship to personality. J. Anat. 224(3): 261-269, 2014.

11. Seagull, F. J., and Rooney, D. M., Filling a void: developing a standard subjective assessment tool for surgical simulation through focused review of current practices. Surgery 156(3):718-722, 2014.

12. Bergman, E. M. et al., How much anatomy is enough? Anat. Sci. Educ. 1:184-188, 2008.

13. Older, J., Anatomy: A must for teaching the next generation. Surgeon 2:79-90, 2004.

14. Cheung, C. L. et al., Use of 3-dimensional printing technology and silicone modeling in surgical simulation: Development and face validation in pediatric laparoscopic pyeloplasty. J Surg Educ. 71: 762-767, 2014.

15. Gottschalk, M. B. et al., Surgical training using three-dimensional simulation in placement of cervical lateral mass screws: A blinded randomized control trial. Spine J. 15:168-175, 2014.

16. Jones, T. W. et al., Use of 3D models of vascular rings and slings to improve resident education. Congenit. Heart Dis. 12(3):578-582, 2017.

17. Garrett, J. et al., Value of 3-D CT in classifying acetabular fractures during orthopedic residency training. Orthopedics 35(5):e615, 2012.

18. Lim, P. K. et al., Use of 3D Printed Models in Resident Education for the Classification of Acetabulum Fractures. J Surg Educ., 2018. 